Article

\title{
Acetamide Derivatives with Antioxidant Activity and Potential Anti-Inflammatory Activity
}

Giuseppina Autore ${ }^{1}$, Anna Caruso ${ }^{1}$, Stefania Marzocco ${ }^{1}$, Barbara Nicolaus ${ }^{2}$, Chiara Palladino ${ }^{1}$, Aldo Pinto ${ }^{1}$, Ada Popolo ${ }^{1}$, Maria S. Sinicropi ${ }^{3}$, Giuseppina Tommonaro ${ }^{2}$ and

Carmela Saturnino ${ }^{1, *}$

1 Department of Pharmaceutical Sciences, University of Salerno, Fisciano (SA), Italy;

E-Mails: autore@unisa.it (G.A.); caruso.anna@inwind.it (A.C.); smarzocco@unisa.it (S.M.); chiara24@hotmail.it (C.P.); pintoal@unisa.it (A.P.); apopolo@unisa.it (A.P.)

2 Institute of Biomolecular Chemistry, CNR, Pozzuoli (NA), Italy;

E-Mails: gtommonaro@icmib.na.cnr.it (G.T.); bnicolaus@icmib.na.cnr.it (B.N.)

3 Department of Pharmaceutical Sciences, University of Cosenza, Arcavacate di Rende (CS), Italy;

E-Mail: s.sinicropi@unical.it (M-S.S.)

* Author to whom correspondence should be addressed; E-Mail: saturnino@unisa.it;

Tel.: +39-89-969769; Fax: +39-89-969602.

Received: 2 February 2010; in revised form: 9 March 2010 / Accepted: 18 March 2010 /

Published: 23 March 2010

\begin{abstract}
This study reports the synthesis and antioxidant activity of some new acetamide derivatives. The compounds' structures were elucidated by NMR analysis and their melting points were measured. The in vitro antioxidant activity of these compounds was tested by evaluating the amount of scavenged ABTS radical and estimating ROS and NO production in $\mathrm{tBOH}$ - or LPS-stimulated J774.A1 macrophages. All compounds were tested for their effect on cell viability by an MTT assay and by a Brine Shrimp Test.
\end{abstract}

Keywords: amides; antioxidant; antiproliferative activity; brine shrimp test

\section{Introduction}

The antioxidant activity is related with compounds capable of protecting a biological system against the potential harmful effects of oxidative processes. In the last years antioxidant compounds have 
received increased attention from nutritionists and medical researchers for their potential activities in preventing cancer, cardiovascular disorders, as well as aging [1]. Several papers have been published on structure-activities analysis of new antioxidant compounds. In this study we report for the first time the synthesis and the in vitro antinflammatory and antioxidant activities of some new acetamide derivatives. The syntheses of the compounds 30000-4 and 6 (as reported in Scheme 1) were accomplished using 2-phenylacetic acid derivatives (1 eq) dissolved in dry dichloromethane (DCM, 10 $\mathrm{mL}$ ). To this solution were added $N, N^{1}$-carbonyldiimidazole (CDI, 2 eq) and 4-dimethylaminopyridine (DMAP, 2 eq) and the mixture was stirred for 20 minutes at rt. After an appropriate 3-phenylpropylamine ( $2 \mathrm{eq}$ ) was added and the mixture further stirred for $5 \mathrm{~h}$ at $\mathrm{rt}$.

Scheme 1. Synthesis of compounds 30000-4 and 6.
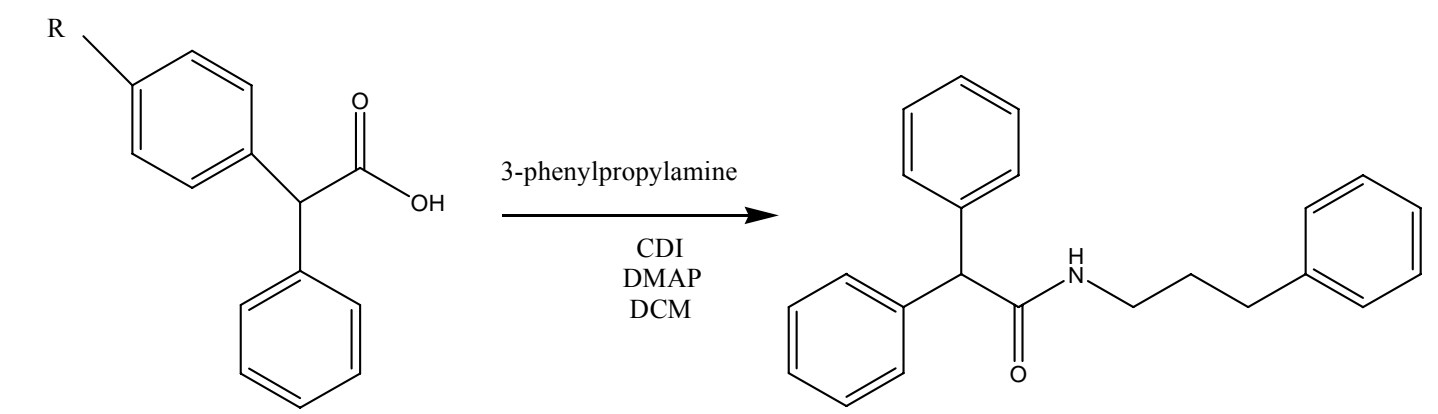

$\mathrm{R}=\mathrm{H}$ 30000; $\mathrm{R}=\mathrm{Cl}$ 30001; $\mathrm{R}=\mathrm{Br} 30002 ; \mathrm{R}=\mathrm{CH}_{3} 30003 ; \mathrm{R}=\mathrm{OCH}_{3} 30004 ; \mathrm{R}=\mathrm{NO}_{2} 30006$.

The synthesis of compound $\mathbf{3 0 0 0 5}$ (Scheme 2) was achieved using 2-(4-methoxyphenyl)-2-phenyl$\mathrm{N}$-(3-phenylpropyl)acetamide (1 eq) and sodium iodide $(2.2 \mathrm{eq})$ dissolved in acetonitrile $(15 \mathrm{~mL})$. Chlorotrimethylsilane ( $2.2 \mathrm{eq}$ ) was added to the solution which was stirred for $16 \mathrm{~h}$ at reflux.

Scheme 2. Synthesis of 2-(4-hydroxyphenyl)-2-phenyl- $N$-(3-phenylpropyl)acetamide (30005).

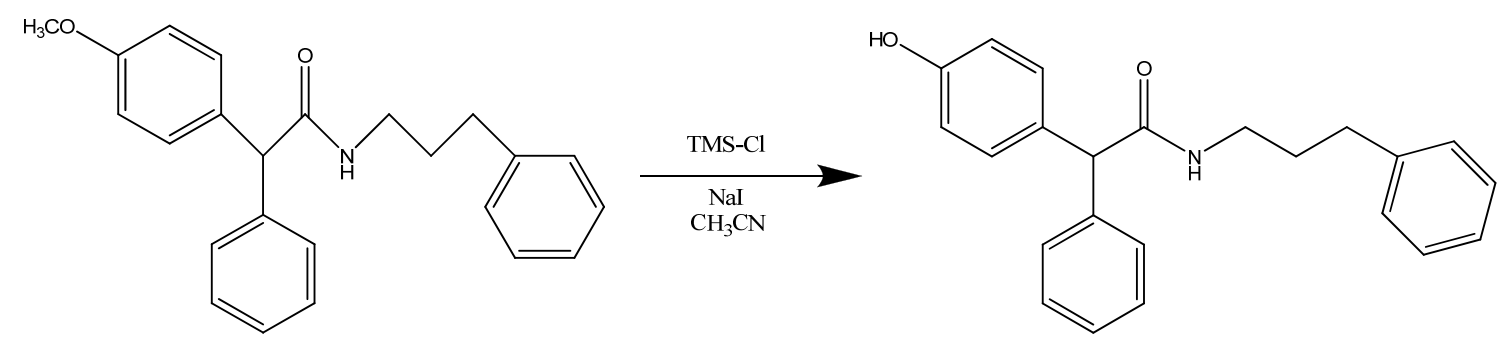

The synthesis of compound $\mathbf{3 0 0 0 7}$ (Scheme 3) was accomplished using 2-(4-nitrophenyl)-2-phenyl$N$-(3-phenylpropyl) acetamide (1 eq) and zinc powder (10 eq) dissolved in EtOH (10 mL). Conc. $\mathrm{HCl}$ ( 1 eq) was added to the solution and cooled to $-10{ }^{\circ} \mathrm{C}$.

The synthesis of the compounds 40000-4 and 6 (Scheme 4) were done using 2-phenylacetic acid derivatives (1 eq) dissolved in dry DCM $(10 \mathrm{~mL})$; afterwards CDI (2 eq) and DMAP (0.5 eq) were added to the solution that was stirred for 20 minutes at rt. Thereafter was added 3,3-diphenylpropan-1amine (1 eq) and the mixture stirred for $24 \mathrm{~h}$ at $\mathrm{rt}$. 
Scheme 3. Synthesis of 2-(aminophenyl)-2-phenyl- $N$-(3-phenylpropyl)acetamide (30007).<smiles>Nc1ccc(C(C(=O)NCCCc2ccccc2)c2ccccc2)cc1</smiles>

Scheme 4. Synthesis of compounds 40000-4 and 6.<smiles>[X]c1ccc(CC(=O)O)cc1</smiles>

$\mathrm{R}=\mathrm{H}$ 40000; $\mathrm{R}=\mathrm{Cl}$ 40001; $\mathrm{R}=\mathrm{Br} 40002 ; \mathrm{R}=\mathrm{CH}_{3} 40003 ; \mathrm{R}=\mathrm{OCH}_{3} 40004 ; \mathrm{R}=\mathrm{NO}_{2} 40006$

The synthesis of compound $\mathbf{4 0 0 0 5}$ (Scheme 5) was achieved using N-(3,3-diphenylpropyl)-2-(4methoxyphenyl)acetamide ( $1 \mathrm{eq})$ and sodium iodide $(3 \mathrm{eq})$ dissolved in acetonitrile $(35 \mathrm{~mL})$. To the solution heated to $23{ }^{\circ} \mathrm{C}$ was added chlorotrimethylsilane (2.2 eq) and the mixture was stirred for $16 \mathrm{~h}$ at reflux.

Scheme 5. Synthesis of $N$-(3,3-diphenylpropyl)-2-(4-hydroxyphenyl)acetamide (40005).<smiles>COc1ccc(CC(=O)NCCC(c2ccccc2)c2ccccc2)cc1</smiles>

The synthesis of compound $\mathbf{4 0 0 0 7}$ (Scheme 6) was accomplished using $N$-(3,3-diphenylpropyl)-2(4-nitrophenyl) acetamide (1 eq) and zinc powder $(10 \mathrm{eq})$ dissolved in $\mathrm{EtOH}(40 \mathrm{~mL})$. Conc. $\mathrm{HCl}$ (1 eq) was added to the solution and it was cooled to $-10^{\circ} \mathrm{C}$.

Scheme 6. Synthesis of 2-(4-aminophenyl)- $N$-(3,3-diphenylpropyl) acetamide (40007).<smiles>Nc1ccc(CC(=O)NCCC(c2ccccc2)c2ccccc2)cc1</smiles> 


\section{Results and Discussion}

The brine shrimp lethality bioassay is an efficient, rapid and inexpensive test developed as a prescreen to evaluate biological activities of natural or synthetic compounds. In Table 1 we report the biological activity of the samples in the brine shrimp assay, expressed as $\mathrm{LD}_{50}$ (in ppm). All compounds showed good activity in this test, in particular compounds 40006 and 40007, which showed interesting $\mathrm{LD}_{50}$ values of 3.0443 and $10.6444 \mathrm{ppm}$, respectively. None of the newly synthesized compounds were cytotoxic on macrophage cell line J774.A1, as revealed by a MTT test (Table 2). Among the tested compounds we choose to further investigate the pharmacological activity of $\mathbf{4 0 0 0 6}$ and $\mathbf{4 0 0 0 7}$ because of their good results in the ABTS and brine shrimp test. LPS increased NO production in the medium of J774.A1 macrophages, addition to the culture medium of graded concentration of 40006 and $40007(0.1-10 \mu \mathrm{M}) 1 \mathrm{~h}$ before and simultaneously to LPS significantly $(P<0.001)$ reduced NO production (Table 3$)$.

Table 1. Biological activity of compounds 30003-30007 and 40003-40007. The experiments were performed in triplicate.

\begin{tabular}{|c|c|c|c|}
\hline SAMPLES & $\%$ INHIBITION ABTS $^{+}$ & T.E.A.C. $(\mu \mathrm{M})$ & $\begin{array}{c}\mathbf{L D}_{\mathbf{5 0}} \mathbf{( p p m )} \\
{[95 \% \text { confidence intervals] }}\end{array}$ \\
\hline 30003 & $1 \%$ & 0.26 & n.a. \\
\hline 30004 & $2 \%$ & 0.52 & n.a. \\
\hline 30005 & $0.8 \%$ & 0.20 & n.a. \\
\hline 30006 & $1 \%$ & 0.26 & $\begin{array}{c}41.4591 \\
{[84.3018 / 23.5460]}\end{array}$ \\
\hline 30007 & $10.6 \%$ & 2.74 & $\begin{array}{c}29.8785 \\
{[45.6981 / 19.8115]}\end{array}$ \\
\hline 40003 & n.a. & I & $\begin{array}{c}58.3200 \\
{[132.3034 / 32.7936]}\end{array}$ \\
\hline 40004 & $2 \%$ & 0.52 & $\begin{array}{c}17.8661 \\
{[28.9967 / 11.1125]}\end{array}$ \\
\hline 40005 & $5.1 \%$ & 1.32 & $\begin{array}{c}17.0035 \\
{[28.5947 / 10.2798]}\end{array}$ \\
\hline 40006 & $4 \%$ & 1.03 & $\begin{array}{c}3.0443 \\
{[4.7830 / 1.9283]}\end{array}$ \\
\hline 40007 & $26.2 \%$ & 6.77 & $\begin{array}{c}10.6444 \\
{[16.8533 / 6.6340]}\end{array}$ \\
\hline
\end{tabular}

Table 2. Shows the results, expressed as IC50 (M) values, indicating the concentration of each compound that affords cell growth by $50 \%$ as compared to control cells. n.d. $=$ not detectable.

\begin{tabular}{|c|c|c|c|}
\cline { 2 - 4 } \multicolumn{1}{c|}{} & \multicolumn{3}{c|}{ Cell lines } \\
\hline Compounds & HEK-293 & WHEI-164 & J774.A1 \\
\hline $\mathbf{3 0 0 0 3}$ & n.d. & n.d. & n.d. \\
$\mathbf{3 0 0 0 4}$ & $1.067 \times 10^{-4}$ & $1.790 \times 10^{-6}$ & n.d. \\
$\mathbf{3 0 0 0 5}$ & $4.170 \times 10^{-5}$ & $2.235 \times \times 10^{-6}$ & n.d.. \\
$\mathbf{3 0 0 0 6}$ & $2.439 \times 10^{-6}$ & $1.913 \times 10^{-4}$ & n.d.. \\
\hline
\end{tabular}


Table 2. Cont.

\begin{tabular}{|c|c|c|c|}
\cline { 2 - 4 } \multicolumn{1}{c|}{} & \multicolumn{3}{c|}{ Cell lines } \\
\hline Compounds & HEK-293 & WHEI-164 & J774.A1 \\
\hline $\mathbf{3 0 0 0 7}$ & n.d. & $1.513 \times 10^{-4}$ & n.d. \\
$\mathbf{4 0 0 0 3}$ & $2.5 \times 10^{-4}$ & $2.441 \times 10^{-6}$ & $>10^{-4}$ \\
$\mathbf{4 0 0 0 4}$ & n.d. & n.d. & $2.324 \times 10^{-4}$ \\
$\mathbf{4 0 0 0 5}$ & $1.665 \times 10^{-4}$ & $1.038 \times 10^{-6}$ & $>10^{-4}$ \\
$\mathbf{4 0 0 0 6}$ & $>10^{-4}$ & $2.05 \times 10^{-5}$ & $2.324 \times 10^{-4}$ \\
$\mathbf{4 0 0 0 7}$ & $2.069 \times 10^{-5}$ & $4.124 \times 10^{-5}$ & $>10^{-4}$ \\
\hline
\end{tabular}

Table 3. Effect of compounds $\mathbf{4 0 0 0 6}$ and $\mathbf{4 0 0 0 7}$ on nitrite production by LPS- induced J774.A1 macrophages. Results are expressed as mean \pm S.E.M. \% inhibition calculated versus nitrite produced by J774.A1 treated with LPS alone of at least 3 independents experiments with three replicates each. Comparison was performed using one way analysis of variance. ${ }^{* *} \mathrm{P}<0.01$ vs. LPS.

\begin{tabular}{|c|c|c|c|}
\cline { 2 - 4 } \multicolumn{1}{c|}{} & \multicolumn{3}{c|}{$\%$ inhibition nitrite production vs LPS } \\
\hline Compounds & $\mathbf{1 0} \boldsymbol{\mu M}$ & $\mathbf{1} \boldsymbol{\mu M}$ & $\mathbf{0 . 1} \boldsymbol{\mu M}$ \\
\hline $\mathbf{4 0 0 0 6}$ & $79.66 \pm 1.33^{* *}$ & $86.75 \pm 0.65^{* *}$ & $91.73 \pm 1.59^{* *}$ \\
$\mathbf{4 0 0 0 7}$ & $85.00 \pm 3.51^{* *}$ & $89.6 \pm 1.46^{* *}$ & $81.5 \pm 12.67 * *$ \\
\hline
\end{tabular}

As reported in Figure 1, compound 40006 also significantly $(\mathrm{P}<0.01)$ reduced ROS production in J774.A1 macrophages stimulated with terz-butyl hyproperoxide ( $\mathrm{tBOH})$, while compound $\mathbf{4 0 0 0 7}$ have no effect on ROS inhibition.

Figure 1. Effect of compounds $\mathbf{4 0 0 0 6}$ and $\mathbf{4 0 0 0 7}$ on ROS production by tBOH-induced J774.A1 macrophages. ROS reaction was determined by measuring the converting reaction of $\mathrm{DCFH}_{2}$ to DCF. ROS production were expressed as mean \pm S.E.M. of relative fluorescent units (RFU) of at least three independent experiments. ${ }^{*} * \mathrm{P}<0.01 \mathrm{vs.} \mathrm{tBOH}$.

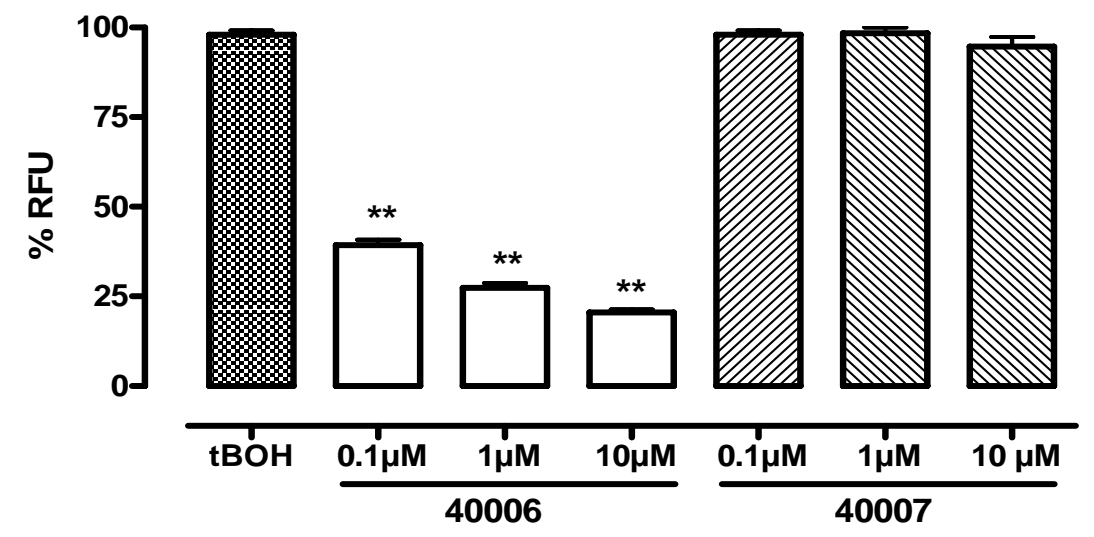

\section{Experimental}

\section{General}

All reagents were purchased from Sigma-Aldrich s.r.l. (Milan, Italy). The reactions were monitored by thin-layer chromatography (TLC), using Whatman K6F silica gel on aluminum and alumina 
(Merck) plates with fluorescence indicators and appropriate solvents. A $>95 \%$ purity could be inferred from the ${ }^{1} \mathrm{H}-\mathrm{NMR}$ spectra. Melting points were taken on a Gallenkamp melting point apparatus and are uncorrected. The organic extracts were dried over sodium sulphate dry (Merck). ${ }^{1} \mathrm{H}-\mathrm{NMR}$ spectra were recorded with a Bruker Advance $300 \mathrm{MHz}$ spectrometer, using $\mathrm{CDCl}_{3}$ as solvent. Mass spectrometry analysis ESI-MS was cwrried out on a Finningan LCQ Deca ion trap instrument.

\section{Synthesis of compounds 30000-4 and $\mathbf{6}$}

An appropriate acid was dissolved in dry DCM $(10 \mathrm{~mL})$. To this solution were added $N, N^{1}$ carbonyldiimidazole (CDI, $0.612 \mathrm{~g} ; 3.78 \mathrm{mmol}$; FW = 162.15; 2 eq) and 4-dimethylaminopyridine (DMAP, $0.115 \mathrm{~g} ; 0.94 \mathrm{mmol} ; \mathrm{FW}=122.17 ; 2 \mathrm{eq}$ ) and the resulting mixture was stirred for 20 minutes at rt. After 3-phenylpropylamine $(0.511 \mathrm{~g} ; 3.78 \mathrm{mmol} ; \mathrm{FW}=135.21 ; 2 \mathrm{eq})$ was added and the mixture stirred for $5 \mathrm{~h}$ at $\mathrm{rt}$ [2]. To the mixture was added $\mathrm{H}_{2} \mathrm{SO}_{4}(2 \mathrm{~N})$ and it was extracted with ethyl acetate. The organic layer was dried with anhydrous sodium sulphate, filtered and the solvent removed by evaporation. The residue was purified by column chromatography on silica (Pe/EtOAc 6:4;Pe/EtOAc 4:6). After recrystallization from DCM/Pe, the products were obtained as white crystals

Compound 30000: Yield: $0.390 \mathrm{~g}(64 \%)$; Melting point: $135{ }^{\circ} \mathrm{C}$; MS: m/z $331\left(\mathrm{M}^{+}+2\right) ; 329\left(\mathrm{M}^{+}\right) ;{ }^{1} \mathrm{H}-$ NMR $\delta 8.0(\mathrm{~s}, 1 \mathrm{H}), 7.39-6.94(\mathrm{~m}, 15 \mathrm{H}), 5.58-5.43(\mathrm{~s}, 1 \mathrm{H}), 3.53-3.30(\mathrm{t}, 2 \mathrm{H}), 2.68-2.56(\mathrm{t}, 2 \mathrm{H}), 1.89-$ $1.77(\mathrm{q}, 2 \mathrm{H})$.

Compound 30001: Yield: $0.650 \mathrm{~g}(70 \%)$; Melting point: $125{ }^{\circ} \mathrm{C}$; MS: $[\mathrm{M}]=363.9 ;\left[\mathrm{M}+\mathrm{H}^{+}\right]=364.9$; ${ }^{1} \mathrm{H}-\mathrm{NMR} \delta$ 7.39-7.04 (m, $\left.14 \mathrm{H}\right), 5.60-5.53(\mathrm{~s}, 1 \mathrm{H}), 4.88(\mathrm{~s}, 1 \mathrm{H}), 3.53-3.37(\mathrm{q}, 2 \mathrm{H}), 2.68-2.60(\mathrm{t}, 2 \mathrm{H})$, $1.89-1.77(\mathrm{q}, 2 \mathrm{H}$

Compound 30002: Yield: $0.210 \mathrm{~g}(81 \%)$; Melting point: $135^{\circ} \mathrm{C}$; $\mathrm{MS}$ : $\left[\mathrm{M}-\mathrm{H}^{+}\right]=407.1$; $\left[\mathrm{M}+\mathrm{H}^{+}\right]=$ 409.1; ${ }^{1} \mathrm{H}-\mathrm{NMR} \delta$ 7.50-7.46 (d, 2H), 7.37-7.10 (m, $\left.12 \mathrm{H}\right), 5.57$ (s, $\left.1 \mathrm{H}\right), 4.48$ (s, $\left.1 \mathrm{H}\right), 3.40-3.30$ (q, $2 \mathrm{H}), 2.64-2.59(\mathrm{t}, 2 \mathrm{H}), 1.96-1.82(\mathrm{p}, 2 \mathrm{H})$.

Compound 30003: Yield: $0.640 \mathrm{~g}(71 \%)$; Melting point: $110^{\circ} \mathrm{C}$; MS: (M) 342.2, $\left(\mathrm{m}+\mathrm{H}^{+}\right) 345.2 ;{ }^{1} \mathrm{H}-$ NMR $\delta$ 7.62-7.21 (m, 12 H), 7.18-7.10 (d, 2 H), 5.57 (s, 1 H), 4.90 (s, 1H), 3.38-3.30 (q, 2 H), 2.632.57 (t, $2 \mathrm{H}), 1.88-1.77$ (p, $2 \mathrm{H})$.

Compound 30004: Yield: 0.640 g (78\%); Melting point: $110{ }^{\circ} \mathrm{C}$; $\mathrm{MS}:[\mathrm{M}]=359.5 ;\left[\mathrm{M}+\mathrm{H}^{+}\right]=360.5$; ${ }^{1} \mathrm{H}-\mathrm{NMR} \delta$ 7.64-7.20 (m, 12H), $6.92(\mathrm{~d}, 2 \mathrm{H}), 5.58(\mathrm{~s}, 1 \mathrm{H}), 4.88(\mathrm{~s}, 1 \mathrm{H}), 3.87$ (s, $\left.3 \mathrm{H}\right), 3.40-3.30$ (q, 2 H), 2.64-3.57 (t, 2 H), 1.89-1.76 (p, 2 H).

Compound 30006: Yield: $0.600 \mathrm{~g}(78 \%)$; Melting point: $117^{\circ} \mathrm{C}$; $\mathrm{MS}:[\mathrm{M}]=374.4 ;\left[\mathrm{M}+\mathrm{H}^{+}\right]=375.4$; ${ }^{1} \mathrm{H}-\mathrm{NMR} \delta 8.40(\mathrm{~d}, 2 \mathrm{H}) ; 7.70(\mathrm{~d}, 2 \mathrm{H}) ; 7.45-7.10(\mathrm{~m}, 10 \mathrm{H}) ; 5.5(\mathrm{~s}, 1 \mathrm{H}) ; 4.87(\mathrm{~s}, 1 \mathrm{H}) ; 3.92-3.85(\mathrm{t}, 2 \mathrm{H})$; $3.35-3.28(\mathrm{q}, 2 \mathrm{H}) ; 2.34-2.22(\mathrm{q}, 2 \mathrm{H})$. 


\section{Synthesis of compounds $\mathbf{3 0 0 0 5}$ and $\mathbf{3 0 0 0 7}$}

2-(4-Methoxyphenyl)-2-phenyl-N-(3-phenylpropyl)acetamide (0.480 g; $1.34 \mathrm{mmol} ; \mathrm{FW}=359.5 ; 1$ eq) and sodium iodide $(0.440 \mathrm{~g} ; 2.95 \mathrm{mmol} ; \mathrm{FW}=149.89 ; 2.2 \mathrm{eq})$ were dissolved in acetonitrile $(15 \mathrm{~mL})$. Chlorotrimethylsilane $(377 \mu \mathrm{L} ; 2.95 \mathrm{mmol} ; \mathrm{FW}=108.64 ; \mathrm{d}=0.850 ; 2.2 \mathrm{eq})$ was added to the solution and stirred for $16 \mathrm{~h}$ at reflux. The mixture was poured into water and extracted with ethyl acetate, the organic layer was washed with $\mathrm{Na}_{2} \mathrm{~S}_{3} \mathrm{O}_{3}$ and Brine, dried with anhydrous sodium sulphate, filtered and evaporated. The residue products were purified by column chromatography on silica (Pe/EtOAc 8:2; Pe/EtOAc 7:3). After recrystallization with DCM/Pe , white crystals were obtained (0.310 g; 66\%). Melting point: $85{ }^{\circ} \mathrm{C} . \mathrm{m} / \mathrm{z} 347.2\left(\mathrm{M}^{+}+2\right) ; 345.2\left(\mathrm{M}^{+}\right) .{ }^{1} \mathrm{H}$ NMR $\delta 8.80-7.18(\mathrm{~m}, 10 \mathrm{H})$; 7.10-7.00 (d, 2H); 6.90-6.77 (d, 2H); $5.67(\mathrm{~s}, 1 \mathrm{H}) ; 4.82(\mathrm{~s}, 1 \mathrm{H}) ; 3.89-3.80(\mathrm{t}, 1 \mathrm{H}) ; 3.33-3.24(\mathrm{q}, 2 \mathrm{H})$; 2.30-2.20 (q, 2H) (30005) .

2-(4-nitrophenyl)-2-phenyl-N-(3-phenylpropyl) acetamide (0.200 g; $0.53 \mathrm{mmol} ; \mathrm{FW}=450.5 ; 1 \mathrm{eq})$ and zinc powder (0.346 g; $5.3 \mathrm{mmol} ; \mathrm{FW}=63.93 ; 10 \mathrm{eq})$ were dissolved in $\mathrm{EtOH}(10 \mathrm{~mL})$. $\mathrm{HCl}$ conc. ( $3 \mathrm{~mL} ; 0.53 \mathrm{mmol} ; \mathrm{FW}=36.46 ; 1 \mathrm{eq}$ ) was added to the solution and cooled to $-10{ }^{\circ} \mathrm{C}$. The mixture was stirred and heated at $80{ }^{\circ} \mathrm{C}$ for $3 \mathrm{~h}$. After was poured into water and $\mathrm{NaOH}$ to neutralize the excess of acid, the organic layer was extracted with $\mathrm{Et}_{2} \mathrm{O}$, dried with anhydrous sodium sulphate, filtered and removed by evaporation. The residue products were purified by column chromatography on silica (Pe/EtOAc 1:1). A yellow-orange oil was obtained $(0.160 \mathrm{~g} ; 88 \%) . \mathrm{m} / \mathrm{z} 346.4\left(\mathrm{M}^{+}+2\right) ; 344.4\left(\mathrm{M}^{+}\right) .{ }^{1} \mathrm{H}$ NMR $\left(\mathrm{CDCl}_{3}, 300 \mathrm{Mhz}\right) \delta$ 7.37-7.19 (m, 8H); $7.15(\mathrm{~d}, 2 \mathrm{H}) ; 7.05(\mathrm{~d}, 2 \mathrm{H}), 6.68(\mathrm{~d}, 2 \mathrm{H}) ; 5.59$ (s, 1H); $4.48(\mathrm{~s}, 1 \mathrm{H}) ; 3.68$ (s, 2H); 3.36 (q, 2H); 2.63 (t, 2H); 2.10 (p, 2H) (30007).

\section{Synthesis of compounds 40000-4 and $\mathbf{4 0 0 0 6}$}

An appropriate acid was dissolved in DCM dry $(10 \mathrm{~mL})$ and $\mathrm{N}^{1}$-carbonyldiimidazole CDI (0.541 g; $4.43 \mathrm{mmol} ; \mathrm{FW}=162.15 ; 2 \mathrm{eq})$ and 4-dimethylaminopyridin $\operatorname{DMAP}(0.134 ; 1.10 \mathrm{mmol} ; \mathrm{FW}=$ $122.17 ; 0.5 \mathrm{eq})$ were added to the solution and stirred for 20 minutes at rt. Thereafter was added 3,3diphenylpropan-1-amine $(0.936 \mathrm{~g} ; 4.43 \mathrm{mmol} ; \mathrm{FW}=211.3 ; 1 \mathrm{eq})$ and stirred for $24 \mathrm{~h}$ at $\mathrm{rt}$. To the mixture was added $\mathrm{H}_{2} \mathrm{SO}_{4}(2 \mathrm{~N})$ and extracted with ethyl acetate. The organic layer was dried with anhydrous sodium sulphate, filtered and removed by evaporation. The residue products were purified by column chromatography on silica (Pe/EtOAc 9:1; Pe/EtOAc 8:2; Pe/EtOAc 7:3; Pe/EtOAc 6:4). After recrystallization with DCM/Pe, white crystals were obtained $(0.620 \mathrm{~g} ; 85 \%)$. [M] $=329 ;\left[\mathrm{M}+\mathrm{H}^{+}\right]$ = 330. ${ }^{1} \mathrm{H}$ NMR $(\mathrm{CDCl} 3,300 \mathrm{Mhz}) \delta 8.0(\mathrm{~s}, 1 \mathrm{H}), 7.41-7.06(\mathrm{~m}, 15 \mathrm{H}), 4.10-4.05(\mathrm{t}, 1 \mathrm{H}), 3.44(\mathrm{~s}, 2 \mathrm{H})$, 3.33-3.20 (t, 2H), 2.19-2.11 (q, 2H) (40000).

After recrystallization with DCM/Pe, white crystals were obtained $(0.750 \mathrm{~g} ; 71 \%)$. [M] $=363$; $\left[\mathrm{M}+\mathrm{H}^{+}\right]=364 .{ }^{1} \mathrm{H}$ NMR $\left(\mathrm{CDCl}_{3}, 300 \mathrm{Mhz}\right) \delta$ 7.64-6.94 (m, 14H), $5.27(\mathrm{~s}, 1 \mathrm{H}), 3.91(\mathrm{t}, 1 \mathrm{H}), 3.47(\mathrm{~s}$, 2H), 3.27-3.16 (t, 2H), 2.29-2.18 (q, 2H) (40001).

After recrystallization with DCM/Pe, white crystals were obtained $(0.700 \mathrm{~g} ; 75 \%)$. [M] $=407$; $[\mathrm{M}+$ $\left.\mathrm{H}^{+}\right]=408 .{ }^{1} \mathrm{H}$ NMR $\left(\mathrm{CDCl}_{3}, 300 \mathrm{Mhz}\right) \beta 7.80(\mathrm{~d}, 2 \mathrm{H}), 7.32-7.12(\mathrm{~m}, 10 \mathrm{H}), 6.96(\mathrm{~d}, 2 \mathrm{H}), 5.33(\mathrm{~s}, 1 \mathrm{H})$, $3.90(\mathrm{t}, 1 \mathrm{H}), 3.46(\mathrm{~s}, 2 \mathrm{H}), 3.27-3.16(\mathrm{t}, 2 \mathrm{H}), 2.29-2.19(\mathrm{q}, 2 \mathrm{H}) \mathbf{( 4 0 0 0 2})$.

After recrystallization with DCM/Pe, white crystals were obtained ( $0.930 \mathrm{~g} ; 82 \%)$. Melting point: $110{ }^{\circ} \mathrm{C} .[\mathrm{M}]=343.5 ;\left[\mathrm{M}+\mathrm{H}^{+}\right]=344.5 .{ }^{1} \mathrm{H} \mathrm{NMR}\left(\mathrm{CDCl}_{3}, 300 \mathrm{Mhz}\right) \delta 7.30-7.16(\mathrm{~m}, 14 \mathrm{H}), 5.30(\mathrm{~s}, 1 \mathrm{H})$, $3.87(\mathrm{t}, 1 \mathrm{H}), 3.50$ (s, 2H), $3.21(\mathrm{q}, 2 \mathrm{H}), 2.25$ (q, 2H) (40003). 
After recrystallization with DCM/Pe, white crystals were obtained $(80 \%) .[\mathrm{M}]=343.5 ;\left[\mathrm{M}+\mathrm{H}^{+}\right]=$ 344.5. ${ }^{1} \mathrm{H}$ NMR $\left(\mathrm{CDCl}_{3}, 300 \mathrm{Mhz}\right) \delta 7.30-7.16(\mathrm{~m}, 14 \mathrm{H}), 5.30(\mathrm{~s}, 1 \mathrm{H}), 3.87(\mathrm{t}, 1 \mathrm{H}), 3.50(\mathrm{~s}, 2 \mathrm{H}), 3.21$ (q, 2H), 2.25 (q, 2H) (40004).

After recrystallization with DCM/Pe, white crystals were obtained $(80 \%)$. $[\mathrm{M}]=343.5 ;\left[\mathrm{M}+\mathrm{H}^{+}\right]=$ 344.5. ${ }^{1} \mathrm{H}$ NMR $\left(\mathrm{CDCl}_{3}, 300 \mathrm{Mhz}\right) \delta$ 7.30-7.16 (m, 14H), $5.30(\mathrm{~s}, 1 \mathrm{H}), 3.87(\mathrm{t}, 1 \mathrm{H}), 3.50(\mathrm{~s}, 2 \mathrm{H}), 3.21$ $(\mathrm{q}, 2 \mathrm{H}), 2.25(\mathrm{q}, 2 \mathrm{H}) \mathbf{( 4 0 0 0 6 )}$.

\section{Synthesis of compounds $\mathbf{4 0 0 0 5}$ and $\mathbf{4 0 0 0 7}$}

$\mathrm{N}$-(3,3-diphenylpropyl)-2-(4-methoxyphenyl)acetamide (1.2 g; $3.4 \mathrm{mmol} ; \mathrm{FW}=359.5 ; 1 \mathrm{eq})$ and sodium iodide ( $1.5 \mathrm{~g} ; 10.2 \mathrm{mmol} ; \mathrm{FW}=149.89 ; 3 \mathrm{eq})$ were dissolved in acetonitrile $(35 \mathrm{~mL})$. To the solution heated to $23{ }^{\circ} \mathrm{C}$ was added chlortrimethylsilane $(1.1 \mathrm{~mL} ; 10.2 \mathrm{mmol} ; \mathrm{FW}=108.64 ; \mathrm{d}=0.850$; $2.2 \mathrm{eq}$ ) and stirred for $16 \mathrm{~h}$ at reflux. The mixture was poured into water and extracted with ethoxyethane, the organic layer was washed with $\mathrm{Na}_{2} \mathrm{~S}_{3} \mathrm{O}_{3}$ and Brine, dried with anhydrous sodium sulphate, filtered and evaporated. The residue products were purified by column chromatography on silica (Pe/EtOAc 8:2; Pe/EtOAc 7:3; Pe/EtOAc 6:4). After recrystallization with DCM/Pe, white crystals were obtained $(0.510 \mathrm{~g} ; 45 \%)$. Melting point: $85^{\circ} \mathrm{C} . \mathrm{m} / \mathrm{z} 347.2\left(\mathrm{M}^{+}+2\right) ; 345.2\left(\mathrm{M}^{+}\right) .{ }^{1} \mathrm{H}$ NMR $\left(\mathrm{CDCl}_{3}, 300 \mathrm{Mhz}\right) \delta 8.80-7.18(\mathrm{~m}, 10 \mathrm{H}), 7.10-7.00(\mathrm{~d}, 2 \mathrm{H}), 6.90-6.77(\mathrm{~d}, 2 \mathrm{H}), 5.67(\mathrm{~s}, 1 \mathrm{H}), 4.82(\mathrm{a}$, $1 \mathrm{H}), 3.89-3.80(\mathrm{t}, 1 \mathrm{H}), 3.33-3.24$ (q, 2H), 2.30-2.20 (q, 2H) (40005).

$\mathrm{N}$-(3,3-diphenylpropyl)-2-(4-nitrophenyl) acetamide (1.00 g; $2.67 \mathrm{mmol} ; \mathrm{FW}=374.4 ; 1 \mathrm{eq})$ and zinc powder $(1.75 \mathrm{~g} ; 26.7 \mathrm{mmol} ; \mathrm{FW}=65.41 ; 10 \mathrm{eq})$ were dissolved in EtOH (40 mL). $\mathrm{HCl}$ conc. ( $5 \mathrm{~mL} ; 0.53 \mathrm{mmol} ; \mathrm{FW}=36.46$; $1 \mathrm{eq}$ ) was added to the solution and cooled to $-10{ }^{\circ} \mathrm{C}$. The mixture was stirred and heated at $80{ }^{\circ} \mathrm{C}$ for $3 \mathrm{~h}$. Thereafter was poured into water and $\mathrm{NaOH}$ to neutralize the excess of acid, the organic layer was extracted with $\mathrm{Et}_{2} \mathrm{O}$, dried with anhydrous sodium sulphate, filtered and removed by evaporation. The residue products were purified by column chromatography on silica (Pe/EtOAc 1:1). Was obtained a yellow-orange oil $(0.900 \mathrm{~g} ; 97 \%)$. m/z $346.4\left(\mathrm{M}^{+}+2\right) ; 344.4$ $\left(\mathrm{M}^{+}\right) .{ }^{1} \mathrm{H}$ NMR $\left(\mathrm{CDCl}_{3}, 300 \mathrm{Mhz}\right), \delta$ 7.37-7.19 (d, 2H), $7.15(\mathrm{~d}, 2 \mathrm{H}), 7.05(\mathrm{~m}, 8 \mathrm{H}), 6.68(\mathrm{~d}, 2 \mathrm{H}), 5.59$ $(\mathrm{s}, 1 \mathrm{H}), 4.84(\mathrm{~s}, 1 \mathrm{H}), 3.68(\mathrm{~s}, 2 \mathrm{H}), 3.36(\mathrm{q}, 2 \mathrm{H}), 2.63(\mathrm{t}, 2 \mathrm{H}), 2.10$ (q, 2H) (40007).

\section{Antioxidant activity assay by ABTS method}

The antioxidant activity of compounds was determined by ABTS method, as previously described [3-4]. This method is based on the capacity of different components to scavenge the ABTS radical cation $\left(\mathrm{ABTS}^{\circ}+\right.$ ) compared to standard antioxidant (Trolox) in dose response curves. Briefly $\mathrm{ABTS}^{*}+$ radical cation is obtained by reacting ABTS $(7 \mathrm{mM})$ with potassium persulphate; this mixture was stored in the dark for 12-16 $\mathrm{h}$ before use. Before the assay, the mixture was diluted in ethanol at a ratio $1: 100$ to give an absorbance at $\lambda=734 \mathrm{~nm}$ of $0.70+0.02$. TROLOX was used as standard at a concentration of $1 \mathrm{mg} / \mathrm{mL}$ and aliquots of Trolox $(0.5 \mu \mathrm{L}, 1 \mu \mathrm{L}, 2 \mu \mathrm{L}, 3 \mu \mathrm{L}, 5 \mu \mathrm{L}$ and $10 \mu \mathrm{L})$ were added to $1 \mathrm{~mL}$ of ethanolic $\mathrm{ABTS}^{\circ}+$ to have a standard curve to which all data are referred. All compounds were dissolved in dichloromethane at a concentration of $20 \mathrm{mg} / \mathrm{mL}$ and $5 \mu \mathrm{L}$ were added to ethanolic $\mathrm{ABTS}^{\circ}+$ to measure absorbance after $1 \mathrm{~min}$.

Antioxidant activity was carried out in triplicate and expressed as percentage of the absorbance of the uninhibited radical solution according to the equation: 


$$
\% \text { inhibition }(\lambda=734 \mathrm{~nm})=\left(1-\mathrm{Abs}_{\mathrm{c}} / \mathrm{Abs}_{0}\right) \times 100
$$

where $\mathrm{Abs}_{0}$ was the absorbance of uninhibited radical solution and $\mathrm{Abs}_{\mathrm{c}}$ is the absorbance measured $1 \mathrm{~min}$ after addition of compound to assay. The antioxidant activity of samples was expressed also as T.E.A.C. (Trolox Equivalent Antioxidant Capacity - $\mu \mathrm{M}$ ) [5].

\section{Brine Shrimps Test}

The brine shrimps (Artemia salina) assay was performed in triplicate with appropriate amounts of samples dissolved in DMSO to reach final concentrations of 1, 10 and $100 \mathrm{ppm}$, using 10 freshly hatched larvae suspended in $5 \mathrm{~mL}$ of artificial sea water [6]. Briefly, for each dose tested, surviving shrimps were counted after $24 \mathrm{~h}$, and the data analyzed by the Finney program [7], which affords $\mathrm{LD}_{50}$ values with $95 \%$ confidence intervals.

\section{Analysis of $\mathrm{NO}_{2}^{-}$Production}

The murine macrophage cell line, J774.A1, was obtained from American Tissue Culture Collection (ATCC). J774.A1 cells were maintained in DMEM supplemented with $\mathrm{NaHCO}_{3}(42 \mathrm{mM})$, hepes (25 mM), penicillin (100 units/mL), streptomycin (100 units/mL), glutamine $(2 \mathrm{mM})$ and foetal calf serum (FCS, $10 \%$ ) at $37{ }^{\circ} \mathrm{C}$ in a $95 \%$ air and $5 \% \mathrm{CO}_{2}$ atmosphere.

J774.A1 $\left(3.5 \times 10^{4}\right.$ cells/well $)$ were plated on 96-well microtiter plates and allowed to adhere at $37{ }^{\circ} \mathrm{C}$ in a $5 \% \mathrm{CO}_{2}$ atmosphere for $2 \mathrm{~h}$. To stimulate the expression of the inducible form of nitric oxide synthase (iNOS), E. coli lipopolysaccharide (LPS, $6 \times 10^{3} \mathrm{u} / \mathrm{mL}$ ) was added to fresh culture medium of J774.A1 with graded concentrations of tested compounds (1-100 $\mu \mathrm{g} / \mathrm{mL})$ added $1 \mathrm{~h}$ before and simultaneously with LPS challenge. Nitric oxide release, evaluated as nitrite $\left(\mathrm{NO}_{2}{ }^{-}\right)$accumulation in cell culture medium, was evaluated $24 \mathrm{~h}$ after LPS stimulation by Griess reagent [8].

\section{MTT Assay for Antiproliferative Activity}

Murine macrophage cells (J774.A1) were maintained as previously indicated. Murine fibrosarcoma cells (WEHI-164) were maintained in adhesion on Petri dishes with DMEM supplemented with 10\% heat-inactivated FCS, hepes $(25 \mathrm{mM})$, penicillin $(100 \mathrm{u} / \mathrm{mL})$ and streptomycin $(100 \mu \mathrm{g} / \mathrm{mL})$. Human embryonic kidney cells (HEK-293) were maintained and grown in adhesion on Petri dishes with DMEM supplemented with FCS $(10 \%)$, hepes $(25 \mathrm{mM})$, penicillin $(100 \mathrm{u} / \mathrm{mL})$ and streptomycin (100 units/mL).

J774.A1, WEHI-164 and HEK-293 (3.5 × $10^{4}$ cells/well) were plated on 96-well microtiter plates and allowed to adhere at $37{ }^{\circ} \mathrm{C}$ in a $5 \% \mathrm{CO}_{2}$ atmosphere for $2 \mathrm{~h}$. Thereafter, the medium was replaced with fresh one $(50 \mu \mathrm{L})$ and a $75 \mu \mathrm{L}$ aliquot of each tested compound was added and then the cells incubated for further 72 h. 6-mercaptopurine was used as reference drug. Mitochondrial respiration, an indicator of cell viability, was assessed by the mitochondrial-dependent reduction of [3-(4,5-dimethylthiazol-2-yl)-2,5-phenyl-2H-tetrazolium bromide] (MTT) to formazan and cells viability was assessed accordingly to the method of Mosmann [9]. Briefly $5 \mu \mathrm{L}$ of MTT $(5 \mathrm{mg} / \mathrm{mL})$ were added and the cells were incubated for an additional $3 \mathrm{~h}$. Thereafter, cells were lysed and the dark blue crystals solubilised with $100 \mu \mathrm{L}$ of a solution containing $50 \%$ (v:v) N, N-dimethylformamide, 
$20 \%$ (w:v) SDS with an adjusted pH of 4.5 [10]. The optical density (OD) of each well was measured with a microplate spectrophotometer (Titertek Multiskan MCC/340) equipped with a $620 \mathrm{~nm}$ filter. The viability of each cell line in response to treatment with tested compounds and 6-mercaptopurine was calculated as: $\%$ dead cells $=100-(\mathrm{OD}$ treated/OD control $) \times 100$.

\section{Measurement of Reactive Oxygen Species (ROS)}

The accumulation of ROS was evaluated by means of the probe 2',7'-dichlorofluorescein (DCF) according to the method described by Le Bel et al.[11]. Briefly, J774.A1 cells were seeded at a density of $5 \times 10^{3}$ cells/well into 96-well plates and allowed to adhere for $2 \mathrm{~h}$. After cell adhesion, examined compounds $(1-100 \mu \mathrm{g} / \mathrm{mL})$ were added to the culture medium $24 \mathrm{~h}$ before and always simultaneously to dichlorofluorescein-diacetate. 2',7'-Dichlorofluorescein-diacetate $\left(\mathrm{H}_{2} \mathrm{DCF}-\mathrm{DA}\right.$, Sigma $)$ was added directly to the growth medium at a final concentration of $5 \mu \mathrm{M}$ and the cells incubated for $1 \mathrm{~h}$ at $37^{\circ} \mathrm{C}$. $\mathrm{H}_{2}$ DCF-DA is a non-fluorescent permeant molecule which diffuses passively into cells; the acetates are then cleaved by intracellular esterases to form $\mathrm{H}_{2} \mathrm{DCF}$ which is thereby trapped within the cell. In the presence of intracellular $\mathrm{ROS}, \mathrm{H}_{2} \mathrm{DCF}$ is rapidly oxidized to the highly fluorescent DCF. Therefore, cells were washed twice with phosphate-buffered saline (PBS), placed in fresh medium and treated with terz-butyl hyproperoxide $(\mathrm{tBOH}) 3 \mathrm{mM}$ for $30 \mathrm{~min}$ and than were placed in a fluorescent microplate reader (LS 55 Luminescence Spectrometer; Perkin Elmer, Beaconsfield, Bucks, UK). Fluorescence was monitored using an excitation wavelength of $490 \mathrm{~nm}$ and an emission wavelength of $520 \mathrm{~nm}$. Results were expressed as Relative Fluorescence Units (RFU).

\section{Conclusions}

Results show that these acetamide derivatives, obtained by simple and rapid chemical synthesis, had interesting biological activities and that none of the compounds are toxic on J774.A1, the cell line chosen for the other tests. Among the tested compounds, 40006 showed the more interesting activity because of its inhibition of nitrite and ROS. Taken together, our data let us hypothesized that the inhibitory effect of compound $\mathbf{4 0 0 0 6}$ on $t$ - $\mathrm{BuOH}$-induced $\mathrm{NO}$ formation in J774.A1 macrophages could be due to its free radical scavenger properties. Moreover, to have an antioxidant activity, is possible to modify the aromatic function binding to the amidic group, instead is necessary to keep unvaried the aromatic function binding to the alkylic chain. In fact the compound $\mathbf{4 0 0 0 6}$ is more active than the compound 30006. Further studies need to characterize its pharmacological activity.

\section{References}

1. Ames, B.N.; Shigenaga, M.K.; Hagen, T.M. Oxidants, antioxidants, and the degenerative diseases of aging. Proc. Natl. Acad. Sci. USA 1993, 90, 7915-7922.

2. Urbani, P.; Ramunno, A.; Filosa, R.; Pinto, A.; Popolo, A.; Bianchino, E.; Piotto, S.; Saturnino, C.; De Prisco, R.; Nicolaus, B.; Tommonaro, G. Antioxidant activity of diphenylpropionamide derivatives: Synthesis, biological evaluation and computational analysis. Molecules 2008, 13, 749-761.

3. Miller, J.N.; Sampson, J.; Candeias, L.P.; Bramley, P.M.; Rice-Evans, C.A. Antioxidant activities of carotenes and xantophylls. FEBS Lett. 1996, 384, 240-242. 
4. Miller, J.N.; Rice-Evans, C.A. Factors influencing the antioxidant activity determined by the ABTS+ radical cation assay. Free Rad. Res. 1997, 26, 195-199.

5. Miller, J.N.; Rice-Evans, C.A.; Davies, M.J.; Gopinathan, V.; Milner, A. A novel method for measuring antioxidant capacity and its application to monitoring the antioxidant status in premature neonates. Clini. Sci. 1993, 4, 407-412.

6. Meyer, B.N.; Ferrigni, N.R.; Putnam, J.E.; Jacobsen, L.B.; Nichols, D.E.; McLaughlin. J.L. Brine shrimp: A convenient general bioassay for active plant constituents. Planta Medica 1982, 45, $31-34$.

7. Finney, D.J. Probit Analysis; Cambridge University Press: Cambridge, UK, 1971.

8. Green, L.C.; Wagner, D.A.; Glogowski, J.; Skipper, P.L.; Wishnok, J.S.; Tannenbaum, S.R. Analysis of nitrate nitrite and ${ }^{15} \mathrm{~N}$-nitrite in biological fluids. Anal. Biochem. 1982, 126, 131-138.

9. Mosmann, T. Rapid colorimetric assay for cellular growth and survival: Application to proliferation and cytotoxic assays. J. Immunol. Methods 1983, 65, 55-63.

10. Opipari, A.W., Jr.; Hu, H.M.; Yabkowitz, R.; Dixit, V.M. The A20 zinc finger protein protects cells from tumor necrosis factor cytotoxicity. J. Biol. Chem. 1992, 297, 12424-12427.

11. Le Bel, C.; Ischiropoulos, H.; Bondy, S.C. Evaluation of the probe 2',7'-dichlorofluorescin as an indicator of reactive oxygen species formation and oxidative stress. Chem. Res. Toxicol. 1992, 5, $227-231$.

Sample Availability: Samples of all compounds cited in the text are available from the authors.

(C) 2010 by the authors; licensee Molecular Diversity Preservation International, Basel, Switzerland. This article is an open-access article distributed under the terms and conditions of the Creative Commons Attribution license (http://creativecommons.org/licenses/by/3.0/). 